\title{
Risk of Rupture of Small Anterior Circulation Aneurysms: An Institutional Experience
}

\author{
Hanish Bansal ${ }^{1}$ Ashwani Chaudhary ${ }^{1}$ Rajdeep Chhina ${ }^{2}$ Apinderpreet Singh ${ }^{1}$ Sandeep Kundra ${ }^{3}$ \\ Kavita Saggar ${ }^{2}$ \\ ${ }^{1}$ Department of Neurosurgery, Dayanand Medical College and \\ Hospital, Ludhiana, Punjab, India \\ 2 Department of Radio-Diagnosis, Dayanand Medical College and \\ Hospital, Ludhiana, Punjab, India \\ ${ }^{3}$ Department of Anesthesia, Dayanand Medical College and Hospital, \\ Ludhiana, Punjab, India \\ Indian J Neurosurg 2015;4:132-135. \\ Address for correspondence Dr. Hanish Bansal, MCh, Department of \\ Neurosurgery, Dayanand Medical College and Hospital, 10-B Udham \\ Singh Nagar, Ludhiana, Punjab 141001, India \\ (e-mail: y2khanish@rediffmail.com).
}

\begin{abstract}
Keywords

- subarachnoid hemorrhage

- aneurysm

- unruptured

Great effort has been made in determining aneurysms at a higher risk of rupture because of risks associated both with their rupture and repair as well. The aim of this study was to determine the most common size of ruptured aneurysms in our patient population with ruptured intracranial aneurysms and to review the literature whether there is a critical size and a specific location at which the incidence of rupture of intracranial aneurysm increases. A retrospective analysis of patients with ruptured intracranial aneurysms admitted in a tertiary care hospital in Northern India between January 2011 and December 2014 was done, and size and location of all ruptured aneurysms were recorded. We stratified the incidence of hemorrhage across the following aneurysm size categories: small (10 mm or less), medium (10-15 mm), large $(15-25 \mathrm{~mm})$, and giant $(>25 \mathrm{~mm})$. Our review included 265 patients with ruptured intracranial aneurysms in which 324 aneurysms were identified. In this series, $87.10 \%(231 / 265)$ of the patients had ruptured aneurysms sized less than $10 \mathrm{~mm}$, and 190 out of 265 patients (71.6\%) had ruptured aneurysms which were less than $7 \mathrm{~mm}$. Most ruptured aneurysms were found on the anterior communicating artery (39.6\%). Our study suggested that we need to recommend surgical treatment for even small unruptured aneurysms with irregular shape, especially anterior circulation aneurysms. Most intracranial aneurysms that are located at the anterior communicating artery and middle cerebral artery bifurcation are small and have high chances of rupture and should be considered for treatment.
\end{abstract}

\section{Introduction}

Subarachnoid hemorrhage from ruptured intracranial aneurysms is associated with significant morbidity and mortality and has prompted interest in the repair of unruptured aneurysms. The life-threatening and debilitating sequelae of subarachnoid hemorrhage of ruptured intracranial aneurysms may be prevented if the

received

September 4, 2015

accepted

September 28, 2015

published online

December 16, 2015 
determine the most common size of ruptured aneurysms in our patient population with ruptured intracranial aneurysms and to review the literature whether there is a critical size and a specific location at which the incidence of rupture of intracranial aneurysm increases.

\section{Materials and Methods}

A retrospective analysis of patients with ruptured intracranial aneurysms admitted in a tertiary care hospital in Northern India between January 2011 and December 2014 was done. Size and location of all ruptured aneurysms were recorded. An approval by the institutional review board was taken.

For the size assessment, we used the maximal size of a given aneurysm. The size was measured on threedimensional computed tomography angiography. In patients with multiple aneurysms, the aneurysm that ruptured was determined with the help of standard radiological and clinical methods, such as patterns of blood on a computed tomography scan; the relative size, site, and presence of loculations on an angiogram; and in some cases, the operative findings.

We stratified the incidence of hemorrhage across the following aneurysm size categories: small (10 mm or less), medium (10-15 mm), large (15-25 mm), and giant (> 25 $\mathrm{mm})$.

\section{Results}

Our review included 265 patients with ruptured intracranial aneurysms in which 324 aneurysms were identified. A total of 223 patients were harboring a single aneurysm, while 42 had multiple aneurysms. A relatively large percentage of patients harbored multiple aneurysms. In patients with multiple aneurysms, the clinical and radiological information was considered, and a judgment of the most likely source of hemorrhage was made.

The mean ruptured aneurysm size was $6.39 \mathrm{~mm}$. Ruptured aneurysms were most likely in the region of 2 to $5 \mathrm{~mm}$ (45.20\%), followed by 5 to $10 \mathrm{~mm}$ (41.13\%). In this series, $87.10 \%(231 / 265)$ of the patients had ruptured aneurysms sized less than $10 \mathrm{~mm}$. Also, 45.20\% (120/265) of the patients in our series had ruptured aneurysms sized less than $5 \mathrm{~mm}$ but more than $2 \mathrm{~mm}$. Two patients (0.75\%) had ruptured cerebral aneurysm sized less than $2 \mathrm{~mm}$. A total of 25 patients (9.4\%) harbored aneurysm between 10 and $15 \mathrm{~mm}, 5$ patients (1.8\%) between 15 and $25 \mathrm{~mm}$, and 4 patients (1.5\%) harbored aneurysm greater than $25 \mathrm{~mm}$ (-Table1).

For the location of the diagnosed ruptured aneurysm, 105 instances were on the anterior communicating artery (39.6\%), 47 were on the middle cerebral artery bifurcation (17.7\%), 24 on the posterior communicating artery (9.05\%), followed by 18 aneurysms located in the distal anterior cerebral artery (6.7\%), while $8.3 \%$ occurred in posterior circulation (22 cases). Posterior circulation aneurysms included basilar top (14 cases), vertebral artery (6 cases),
Table 1 Distribution according to sizes

\begin{tabular}{|l|l|}
\hline Size of aneurysm $(\mathrm{mm})$ & Number of cases $(\boldsymbol{n}=265)$ \\
\hline$<5$ & 122 \\
\hline $5-10$ & 109 \\
\hline $10-15$ & 25 \\
\hline $15-25$ & 5 \\
\hline$>25$ & 4 \\
\hline
\end{tabular}

anterior inferior cerebellar artery (1 case), and posterior inferior cerebellar artery ( 1 case). Other locations included anterior choroidal artery (20 cases), internal carotid artery bifurcation ( 9 cases), cavernous sinus, internal carotid artery (9 cases), an M1 segment of middle cerebral artery ( 5 cases), an M2 segment of middle cerebral artery (3 cases), A1 segment of anterior cerebral artery (2 cases), and paraclinoid internal carotid artery (1 case).

Mean size of ruptured anterior communicating artery aneurysm was $5.55 \mathrm{~mm}$, posterior communicating artery aneurysm was $6.32 \mathrm{~mm}$, distal anterior cerebral artery aneurysm was $4.35 \mathrm{~mm}$, and middle cerebral artery bifurcation was $7.06 \mathrm{~mm}$. While the mean size of ruptured internal carotid artery bifurcation aneurysm was $7.52 \mathrm{~mm}$, cavernous internal carotid artery aneurysm was $8.23 \mathrm{~mm}$ and basilar top aneurysm was $6.07 \mathrm{~mm}$. In this series, 42 patients had multiple aneurysms and harbored a total of 101 aneurysms.

\section{Discussion}

Intracranial aneurysm size continues to be an efficient and widely used measure for aneurysmal rupture risk assessment. Currently, aneurysmal risk assessment is mainly based on aneurysm size, that is, smaller aneurysms are less likely to rupture than larger aneurysms.

Multiple studies have attempted to determine the critical size at which aneurysm is likely to rupture, but there is great disagreement in the literature regarding a critical size for rupture of intracranial aneurysm. Some studies emphasize that besides aneurysmal size, other factors such as aneurysm location and multiplicity should also be considered for treatment of unruptured intracranial aneurysms. Size remains the most significant factor of these to stratify the risk of rupture of intracranial aneurysms.

Weir et al in a retrospective analysis of 945 patients with intracranial aneurysms noted that the mean size of all unruptured aneurysms was significantly smaller than the mean size of all ruptured aneurysms $(10.8 \mathrm{~mm})$. Their data confirmed that ruptured aneurysms are usually larger than unruptured aneurysms, and the majority of unruptured aneurysms were smaller than $10 \mathrm{~mm}$. They also noted that $40.3 \%$ of the ruptured aneurysms were on the anterior cerebral artery or anterior communicating artery and none of the cavernous internal carotid artery aneurysms were ruptured. $^{2}$

A prospective study of 5,720 patients with newly diagnosed aneurysms from Japan concluded that cerebral 
aneurysms larger than $7 \mathrm{~mm}$, located in the anterior communicating or internal carotid-posterior communicating arteries and with a daughter sac, are associated with an increased risk of rupture. ${ }^{3}$ In a systematic review, Rinkel et al found out that large majority of aneurysms are small $(<10 \mathrm{~mm})$, and the annual risk of rupture of these small aneurysms is low while symptomatic aneurysms, posterior circulation aneurysms, and large aneurysms ( $>10 \mathrm{~mm}$ ) had a higher risk of rupture. ${ }^{4}$

A prospective study from Finland that followed 118 patients with unruptured intracranial aneurysms until death or subarachnoid hemorrhage found out that the patients with unruptured intracranial aneurysms of $7 \mathrm{~mm}$ or more in size had an increased risk of a lifetime subarachnoid hemorrhage, particularly among women, compared with the patients with smaller unruptured intracranial aneurysms. ${ }^{5}$ A study analyzing risk-benefits of treatment of unruptured intracranial aneurysm concluded that in patients with incidental anterior circulation aneurysms under $7 \mathrm{~mm}$ in diameter repairing the aneurysm brings a slight reduction in life expectancy at all ages; while in all patients with additional aneurysm and those with incidental aneurysms $7 \mathrm{~mm}$ or more in diameter or of the posterior circulation, life years are gained by repair up to the age of 45 to 65 years. $^{6}$

Howsoever, in our series, only 34 patients (12.8\%) had aneurysm size greater than $10 \mathrm{~mm}$. In this series, 190 out of 265 patients $(71.6 \%)$ had ruptured aneurysms, which were less than $7 \mathrm{~mm}$. Also, the most common location for ruptured aneurysm was anterior communicating artery (39.6\%). There is a striking mismatch between our data and the natural history of unruptured intracranial aneurysms as found by the International Study of Unruptured Intracranial Aneurysms (ISUIA) in terms of size of ruptured cerebral aneurysms. Ruptured aneurysms less than $7 \mathrm{~mm}$ located in the anterior circulation constitute the majority of all ruptured intracranial aneurysms found in our retrospective analysis, but unruptured aneurysms less than $7 \mathrm{~mm}$ located at the anterior circulation bear a risk of rupture of $0 \%$ according to the prospective ISUIA data. ${ }^{7}$ Out of 105 , a total of 80 ruptured anterior communicating artery aneurysms (76.1\%) were smaller than $7 \mathrm{~mm}$ in our series.

Komotar et al in 2008 reported that taking $5 \mathrm{~mm}$ as the cutoff for treatment will ensure that $99 \%$ of all aneurysms that should be treated will have treatment offered. It also concluded that small, incidental aneurysms less than $5 \mathrm{~mm}$ should be managed conservatively. ${ }^{8}$ In our study, mean size of ruptured aneurysms according to their locations were $5.55 \mathrm{~mm}$ in the anterior communicating artery, $6.32 \mathrm{~mm}$ in the posterior communicating artery, and $4.35 \mathrm{~mm}$ in the distal anterior cerebral artery. Out of the 47 patients, 31 patients (65.95\%) with ruptured middle cerebral artery bifurcation aneurysms had size less than $7 \mathrm{~mm}$. These results contradict the ISUIA results that small aneurysms are safe.

Forget et al in a review of 362 cases of cerebral aneurysms found that regardless of location on the circle of Willis, $85.6 \%$ of all aneurysms presenting with rupture were less than $10 \mathrm{~mm}$. They argued that the risk of rupture of small intracranial aneurysms is not insignificant, especially those of anterior communicating artery. They also discussed histological and angiographic evidence and refuted the explanation that aneurysms shrink after rupture and hence yield small diameters after rupture. ${ }^{9}$

Beck et al in an analysis of the size and location of 155 saccular intracranial aneurysms in 118 consecutive patients, with the help of three-dimensional rotational angiography, noted that the majority of ruptured aneurysms, 81.9 and $59 \%$, were smaller than 10 and $7 \mathrm{~mm}$; likewise, 81.9 and $68.1 \%$ of unruptured aneurysms were smaller than 10 and $7 \mathrm{~mm}$ and the difference between two groups was not significant. They concluded that small aneurysm (below 7 $\mathrm{mm}$ ), also in the anterior circulation, should be carefully evaluated for treatment. $^{10}$

Al-Jehani et al in a retrospective analysis of 192 patients harboring 213 aneurysms noted that the majority of the diagnosed ruptured aneurysms were smaller than $10 \mathrm{~mm}$ and concluded that decision to treat a given unruptured intracranial aneurysm should be individually assessed and not taken from general international literature as this may mistakenly apply factors from one population to another. ${ }^{11}$

Orz and AlYamany in a study stated that out of ruptured 76 aneurysms, 47 aneurysms (62\%) were smaller than $7 \mathrm{~mm}$ in diameter, and the ruptured anterior communicating artery aneurysms less than $7 \mathrm{~mm}$ in diameter were much more common than aneurysms at any other location indicating that the anterior communicating artery is the most dangerous site for aneurysm rupture. ${ }^{12}$ In this study, 105 patients out of 265 patients (39.6\%) had their ruptured aneurysms located at the anterior communicating artery as compared with other sites.

Rosenørn and Eskesen in Danish Aneurysm Study found that out of 948 ruptured aneurysms verified by angiography, 162 ruptured aneurysms were smaller than $5 \mathrm{~mm}, 474$ and 272 were between 5 and $10 \mathrm{~mm}$ and 11 and $24 \mathrm{~mm}$, respectively. The study concluded that a critical size for unruptured intracranial aneurysms below which there is a benign prognosis cannot be defined because if an intracranial aneurysm ruptures, the consequences are very serious with high mortality and morbidity, also after rupture of the smaller aneurysms. ${ }^{13}$

Tremmel et al in a study suggested that the absolute size of an intracranial aneurysm is less relevant to the development of dangerous intra-aneurysmal hemodynamics than is the relationship between intracranial aneurysm size and parent vessel caliber (quantified by aneurysm to parent vessel size ratio). The study concluded that higher size ratio, irrespective of aneurysm type and absolute aneurysm or vessel size, gives rise to flow patterns typically observed in ruptured intracranial aneurysms. ${ }^{14}$

Rahman et al in a prospective study of 40 consecutive patients with intracranial aneurysms stated that ruptured intracranial aneurysms are larger than unruptured intracranial aneurysms, but size alone may not take into account additional characteristics or hemodynamics that may increase rupture risk. They concluded that size ratio, 
the ratio between aneurysm size and parent artery diameter correlates with an intracranial aneurysm rupture status on presentation in a blinded analysis. ${ }^{15}$

Weir et al in a study highlighted the significance of aspect ratio (dome/neck ratio) and stated that high aspect ratio might reasonably influence the decision to treat actively an unruptured aneurysm independent of its maximum size. ${ }^{16}$ An aneurysm with larger aspect ratio is much more likely to rupture as compared with aneurysm with smaller aspect ratio, even if both have the same maximum diameters. A multicentric study from Japan studying the natural course of aneurysms in 181 cases with 209 aneurysms concluded that the risk of rupture and morbidity in relation to surgical treatment cannot be predicted by size alone and the morphology, especially the presence of blebs, should be considered when treating unruptured cerebral aneurysms. ${ }^{17}$

The role of hemodynamic forces in the initiation, growth, and rupture of cerebral aneurysms have been studied in numerous experimental models and clinical studies. Cebral et al constructed computational fluid dynamics model using three-dimensional rotational angiography imaging data and postulated that simple, stable pattern, large impingement regions, and large jet sizes were more commonly seen with unruptured aneurysms. ${ }^{18}$ Another qualitative hemodynamic analysis of cerebral aneurysms by using image-based patient-specific geometries by Cebral et al concluded that concentrated inflow jets, small impingement regions, complex flow patterns, and unstable flow patterns were correlated with a clinical history of aneurysm rupture. ${ }^{19}$

The limitations of this study include its retrospective nature and a subjective bias in determining aneurysm dimensions.

\section{Conclusion}

Our study suggested that we need to recommend surgical treatment for even small, unruptured aneurysms with irregular shape, especially anterior circulation aneurysms. The decision to treat a given unruptured intracranial aneurysm should be individually assessed and not taken from general international literature. The majority of the intracranial aneurysms that are located at the anterior communicating artery and middle cerebral artery bifurcation are small and have high chances of rupture and should be considered for treatment.

\section{Conflict of Interest}

None.

\section{Source of Support}

None.

Note

This article has been approved by all the authors. This article has not been presented in any conference.

\section{References}

1 Ojini FI. Natural history of cerebral saccular aneurysms. West Afr J Med 2005;24(1):70-74

2 Weir B, Disney L, Karrison T. Sizes of ruptured and unruptured aneurysms in relation to their sites and the ages of patients. J Neurosurg 2002;96(1):64-70

3 Morita A, Kirino T, Hashi K, et al; UCAS Japan Investigators. The natural course of unruptured cerebral aneurysms in a Japanese cohort. N Engl J Med 2012;366(26):2474-2482

4 Rinkel GJ, Djibuti M, Algra A, van Gijn J. Prevalence and risk of rupture of intracranial aneurysms: a systematic review. Stroke 1998;29(1):251-256

5 Korja M, Lehto H, Juvela S. Lifelong rupture risk of intracranial aneurysms depends on risk factors: a prospective Finnish cohort study. Stroke 2014;45(7):1958-1963

6 Vindlacheruvu RR, Mendelow AD, Mitchell P. Risk-benefit analysis of the treatment of unruptured intracranial aneurysms. J Neurol Neurosurg Psychiatry 2005;76(2):234-239

7 Wiebers DO, Whisnant JP, Huston J III, et al; International Study of Unruptured Intracranial Aneurysms Investigators. Unruptured intracranial aneurysms: natural history, clinical outcome, and risks of surgical and endovascular treatment. Lancet 2003;362(9378): $103-110$

8 Komotar RJ, Mocco J, Solomon RA. Guidelines for the surgical treatment of unruptured intracranial aneurysms: the first annual J. Lawrence pool memorial research symposium-controversies in the management of cerebral aneurysms. Neurosurgery 2008; 62(1):183-193, discussion 193-194

9 Forget TR Jr, Benitez R, Veznedaroglu E, et al. A review of size and location of ruptured intracranial aneurysms. Neurosurgery 2001; 49(6):1322-1325, discussion 1325-1326

10 Beck J, Rohde S, Berkefeld J, Seifert V, Raabe A. Size and location of ruptured and unruptured intracranial aneurysms measured by 3dimensional rotational angiography. Surg Neurol 2006;65(1): 18-25, discussion 25-27

11 Al-Jehani H, Najjar A, Sheikh BY. Determining the critical size of intracranial aneurysm predisposing to subarachnoid hemorrhage in the Saudi population. Asian J Neurosurg 2014; 9(4):193-195

12 Orz Y, AlYamany M. The impact of size and location on rupture of intracranial aneurysms. Asian J Neurosurg 2015;10(1):26-31

13 Rosenørn J, Eskesen V. Does a safe size-limit exist for unruptured intracranial aneurysms? Acta Neurochir (Wien) 1993;121(3-4): 113-118

14 Tremmel M, Dhar S, Levy EI, Mocco J, Meng H. Influence of intracranial aneurysm-to-parent vessel size ratio on hemodynamics and implication for rupture: results from a virtual experimental study. Neurosurgery 2009;64(4):622-630, discussion 630-631

15 Rahman M, Smietana J, Hauck E, et al. Size ratio correlates with intracranial aneurysm rupture status: a prospective study. Stroke 2010;41(5):916-920

16 Weir B, Amidei C, Kongable G, et al. The aspect ratio (dome/neck) of ruptured and unruptured aneurysms. J Neurosurg 2003;99(3): 447-451

17 Tsukahara T, Murakami N, Sakurai Y, et al. Treatment of unruptured cerebral aneurysms; a multi-center study at Japanese national hospitals. Acta Neurochir Suppl (Wien) 2005; 94:77-85

18 Cebral JR, Castro MA, Burgess JE, Pergolizzi RS, Sheridan MJ, Putman CM. Characterization of cerebral aneurysms for assessing risk of rupture by using patient-specific computational hemodynamics models. AJNR Am J Neuroradiol 2005;26(10): 2550-2559

19 Cebral JR, Mut F, Weir J, Putman CM. Association of hemodynamic characteristics and cerebral aneurysm rupture. AJNR Am J Neuroradiol 2011;32(2):264-270 